\title{
A New Approach for the Detection of Concrete Cracks Based on Adaptive Morphological Filtering
}

\author{
Huan Hou and Weiguo Lin ${ }^{1}$ \\ College of Information Science and Technology, Beijing University of Chemical \\ Technology, Beijing 100029, China
}

\begin{abstract}
Cracks are an important sign of distress of concrete bridges and may reduce their service life and safety. For the case where there are stains, peeling, scratches, and uneven illumination on the surface of concrete bridges, and where it is difficult to accurately detect complete cracks, this paper proposes a new method to connect the breaks in cracks by adaptive morphological dilation based on crack direction. Most of the existing crack image detection methods attempt to achieve high detection accuracy by increasing the algorithm complexity but sacrifice realtime detection efficiency. A multiple filtering method based on a few adaptive feature thresholds is proposed to filter non-cracks and obtain a clear crack image by analyzing the morphological characteristic differences between real cracks and noise and pseudo-cracks. The experimental results show that the proposed method can effectively improve the integrity of cracks, remove different noise and pseudocracks, without modeling, and has a higher detection accuracy and speed, which is suitable for practical engineering applications.
\end{abstract}

Keywords. concrete crack detection, infrared image, morphology, multiple feature thresholds, adaptive filtering

\section{Introduction}

Bridges play an important role in transportation. Most modern bridges are constructed from reinforced concrete, which can be damaged by natural disasters, environmental temperature, aging of building materials, overload, and other factors. This reduces bridge lifespan, safety, and transport efficiency [1]. Cracks are a common defect in bridges, which belong to the first stage of bridge hazards. They not only affect the normal use of bridges, but also induce other damage [2]. Therefore, crack detection and evaluation of the surface of bridge structures play an important role in the maintenance of the structural health and reliability of concrete bridges [3]. Traditional manual crack detection methods are time-consuming, laborious, dangerous, and subjective, and cannot meet the requirements of high efficiency, automatic detection, and convenient accessibility [4].

${ }^{1}$ Corresponding Author: Weiguo Lin, College of Information Science and Technology, Beijing University of Chemical Technology, Beijing 100029, China; Email: linwg@mail.buct.edu.cn 
The development of high-speed camera technology and large storage hardware makes it straightforward to collect road images in real time. Therefore, image-based technology provides an efficient and economical way to detect cracks. Combined with unmanned aerial vehicles (UAVs) and portable equipment, this area has attracted more and more attention from academic research and industry [5]. Image-based crack detection processing mainly includes image noise reduction, crack extraction, and crack recognition. Su and Yang [6] proposed a morphological segmentation algorithm based on edge detection to realize the automatic detection of cracks on concrete surfaces. In order to make use of the local structural features of cracks, Shi et al. [7] proposed the CrackForest method to detect road cracks automatically based on random structured forests, which can effectively suppress noise. However, this method requires manual threshold selection to segment the image. Zhao et al. [8] regarded each crack gray image as a parametric surface, and cluster the candidate points by geometric representation and anisotropy of cracks to detect inconspicuous cracks. Jahanshahi et al. [9] proposed a non-contact remote sensing crack detection and quantization method based on $3 \mathrm{D}$ scene reconstruction, image processing, and pattern recognition. This method uses depth perception to detect cracks and quantify their width, which has high reliability, but the $3 \mathrm{D}$ image requires more computation and cannot achieve real-time performance.

In order to improve crack detection performance, Lee et al. [10] combine morphology with a neural network to detect, measure, and analyze the width, length, direction, and pattern of cracks. Jang et al. [11] proposed a concrete crack detection approach based on deep learning (DL). This method uses hybrid images, which combine visual and infrared thermal imaging images, and improves the convolutional neural network (CNN) structure, which can automatically detect macro and micro cracks while minimizing the false alarm rate. Nhung et al. [12] applied a CNN to detect cracks in pavement images, achieving high accuracy without image preprocessing. Although the CNN-based method has high detection accuracy, it requires a large number of labeled images including actual crack and non-crack images to support the training process. It also requires a large amount of computation and high-performance hardware, taking a long time to run.

Due to the complex geographical and natural environment and concrete surface roughness, the collected concrete images have problems such as spalling, stains, scratches, and uneven illumination, which affect the detection accuracy. Most crack detection algorithms cannot address these problems simultaneously. Compared with ordinary images, infrared image detection greatly reduces the impact of these problems. Any object produces thermal radiation. The intensity of the infrared radiation of the object is not only related to the material type, morphological characteristics, and chemical and physical structure (such as surface oxidation, roughness, etc.), but is also related to the temperature. In the application of concrete crack detection, by analyzing infrared thermal imaging and comparing the difference of the infrared radiation distribution between the crack area and the surrounding concrete surface, the morphology and location of cracks are determined to achieve the detection of crack defects [13]. Tashan et al. [14] used an active infrared thermal imaging detection method to investigate carbon fiber reinforced polymer concrete samples containing various artificial and loading cracks on the concrete surface. It can detect the location and width of cracks well, but it is affected by the heating angle and position. Jang et al. [15] proposed a line laser thermography scanning system for multiple crack evaluation on a concrete structure, and infrared images are used to successfully visualize and 
evaluate multiple cracks. However, the detection speed is slow, and the system is only designed for use with large laboratory equipment.

In this paper, infrared images are used to detect concrete cracks. Generally, crack detection methods based on image processing often have the problem of crack breaks after threshold segmentation, resulting in low detection accuracy and incomplete cracks detected. This paper proposes a new method of morphological dilation based on the direction of cracks to connect the breaks and improve the integrity of the crack. Most existing crack image detection methods try to achieve higher detection accuracy by increasing the algorithm complexity, but at the same time sacrifice the real-time detection efficiency. Taking into account the difference in the morphological characteristics of the connected domain between cracks and noise, a multi-filtering method based on adaptive feature thresholds is proposed to remove non-cracks and detect more complete cracks. The method does not need to establish a crack diagnosis model, and has a high detection accuracy rate, which not only improves the applicability of the method, but also ensures real-time performance.

\section{Infrared image crack detection method}

In the process of the automatic detection of cracks in concrete bridge images, the collected infrared images have large noise interference due to light, equipment noise, and other reasons. Therefore, it is necessary to preprocess the image first to improve the quality, and then perform image background removal and threshold segmentation. After this, the binary image is subjected to morphological dilation based on the crack direction to improve the integrity of the crack, and finally, adaptive morphological filtering is performed to obtain the detection result. The flow chart of the crack detection method proposed in this paper is shown in Figure 1.

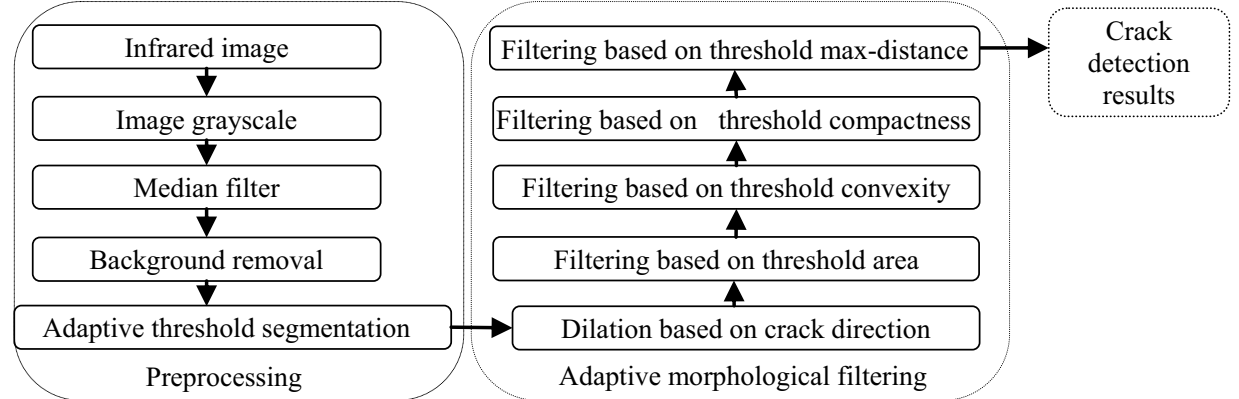

Figure 1. Flow chart of the crack detection processes.

\subsection{Connected domain feature distribution for crack infrared images}

After threshold segmentation, binary images can be obtained. The image domain composed of target pixels with the same pixel value and adjacent positions is called the connected domain. Pixels of the same crack target usually have connectivity. In this paper, by analyzing the differences in the morphological characteristics of the connected domains between cracks, noise, and pseudo-cracks, several connected domain features are proposed, and then the distribution characteristics of the features are analyzed. The features of the crack connected domain used in this paper mainly 
include: (1) The area of the connected domain A; (2) Convexity feature CV; (3) Compactness feature COM; (4) The max distance L in the connected domain [16-19].

Figure 2 shows the binary images with and without cracks after background subtraction and adaptive threshold segmentation [19]. The distributions of the four kinds of features mentioned above are shown in Figure 3(a). It can be seen that the two curves for crack and non-crack images are significantly different because of the influence of the crack features, in which the positions of the crack features are marked with small circles. For the image with cracks, the kernel smoothing density estimation (ksdensity) curves for the four features were obtained and then their normal distribution $N\left(a * \mu_{i},\left(b * \sigma_{i}\right)^{2}\right)$ curves are obtained, as shown in Figure 3(b). It can be seen that for the infrared image with cracks, most of the connected domains are still noise and pseudo-cracks, with only one or a few real cracks. Therefore, the above four features of noise and pseudo-cracks which account for the majority are basically within the range $(\mu-2 \sigma, \mu+2 \sigma)$ of the normal distribution $N\left(a * \mu_{i},\left(b * \sigma_{i}\right)^{2}\right)$, while the features of real cracks (marked by dots) are distributed outside this range.

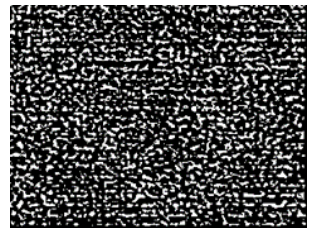

(a) Non-crack image.

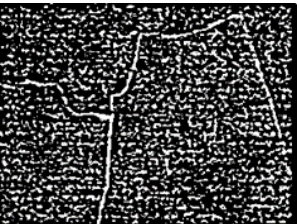

(b) Crack image.

Figure 2. Images after background removal and segmentation.
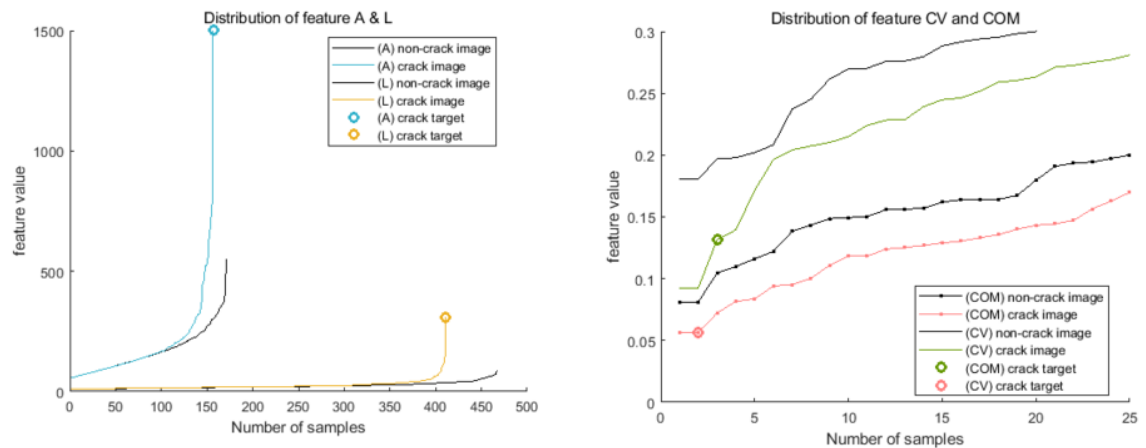

(a) Comparison of images with and without cracks.
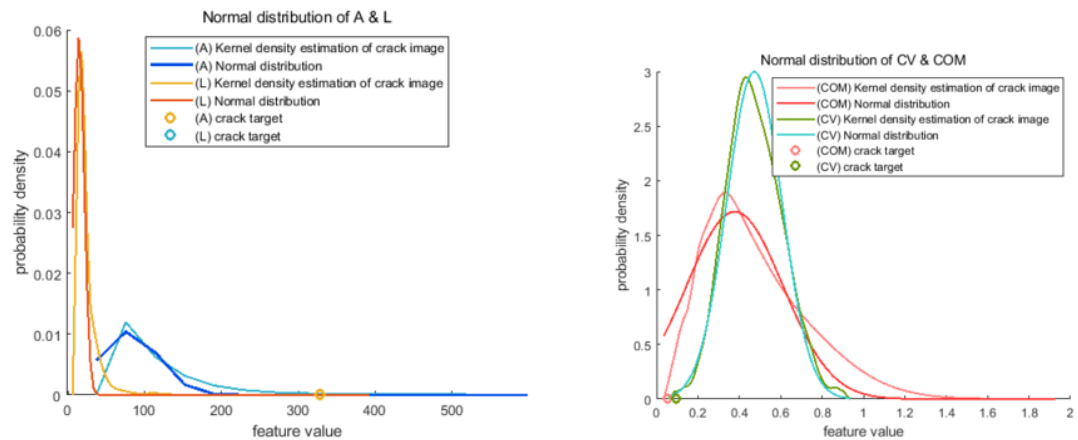

(b) Distribution of crack image features.

Figure 3. Distribution and comparison of connected domain features in infrared images. 
Through the statistical analysis of the experimental results, the parameters $a$ and $b$ that fit the normal distribution can be obtained. Several infrared images with cracks are randomly selected as samples, and experimental statistical analysis is conducted for the four features to obtain the parameters $a$ and $b$ that minimize the mean squared error (MSE) of the fitting normal distribution $N\left(a * \mu_{i},\left(b * \sigma_{i}\right)^{2}\right)$. According to the results, the normal distribution $N\left(\mu, \sigma^{2}\right)$ and the feature value distributions of the four connected domain features in the image are as follows:

(1) $\mathrm{F}(\mathrm{A}) \sim \mathrm{N}\left(0.6 \mu_{1},\left(\sigma_{1} / 4\right)^{2}\right), \mathrm{T}_{\mathrm{A}}>0.6 \mu_{1}+\sigma_{1} / 2$.

(2) $\mathrm{F}(\mathrm{CV}) \sim \mathrm{N}\left(\mu_{2}, \sigma_{2}^{2}\right), \mathrm{T}_{\mathrm{CV}}<\mu_{2}-2 \sigma_{2}$.

(3) $\mathrm{F}(\mathrm{COM}) \sim \mathrm{N}\left(0.6 \mu_{3}, \sigma_{3}^{2}\right), \mathrm{T}_{\mathrm{COM}}<0.6 \mu_{3}-\sigma_{3}$.

(4) $\mathrm{F}(\mathrm{L}) \sim \mathrm{N}\left(0.6 \mu_{4},\left(\sigma_{4} / 4\right)^{2}\right), \mathrm{T}_{\mathrm{L}}>\mu_{4}+\sigma_{4} / 2$.

Here, $\mathrm{T}_{\mathrm{A}}, \mathrm{T}_{\mathrm{CV}}, \mathrm{T}_{\mathrm{COM}}$, and $\mathrm{T}_{\mathrm{L}}$ can be used as the four adaptive crack feature thresholds which are the basis for selecting the target connected domains that conform to crack characteristics in the image. According to these adaptive thresholds, multistage filtering can be performed on noise and pseudo-cracks.

\subsection{Adaptive dilation based on crack direction}

After threshold segmentation, multiple breaks often appear in the image, resulting in incomplete cracks. In the basic operations of morphology, dilation will fill the edge breaks or holes, but usually the dilation operation will connect the target crack with surrounding noise, destroying the real shape of the crack. In this paper, an adaptive dilation method based on the crack direction is proposed, which can enhance the completeness of cracks and ensure that the shape of cracks is unchanged to a certain extent. The method first constructs structural elements that can adapt to the direction of the crack connected domain, which determines the direction of the subsequent dilation operation. Second, only the connected domains conforming to crack characteristics is processed by morphological dilation, while the noise domains are not, which protects the edge of the cracks and also reduces the amount of operations.

(1). For the image $B_{0}$ after threshold segmentation, the adjacent target connected domains $A_{n}$ with larger areas and less compactness are selected, which are more consistent with crack characteristics. An initial horizontal structural element $S_{0}$ is constructed with a length of 12 pixels, as shown in Figure 4(a). ( $n$ represents the $n$-th target connected domain.)

(2). The angle of domain $A_{n}$ is $\theta_{n}$,which is the angle between the major axis of the same standard second order center distance ellipse and the positive $x$-axis. $\mathrm{S}_{0}$ rotates at the angle of $\theta_{n}$ to construct the adaptive structural element $S_{n}$, so $S_{n}$ is in the same direction as $A_{n}$. Figure $4($ b) shows examples of a structural element rotated at different angles.

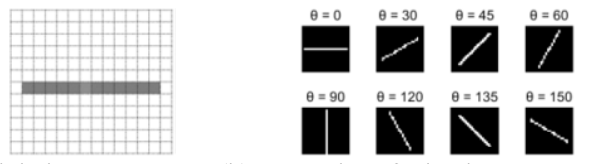

(a) Initial element.

(b) Examples of adaptive structural element $\boldsymbol{S}_{\boldsymbol{n}}$.

Figure 4. Construction of adaptive structural elements with angles of $\theta_{n}$.

(3). The structural element $S_{n}$ is used for adaptive morphological dilation with the domain $A_{n}$; the result is fused with the original image $B_{0}$ to obtain whole image $B_{n}$. 
(4). Steps 2 and 3 are repeated until the adaptive dilation of all target domains is completed; the final image $B_{N}$ is obtained, as shown in Eq. (1).

$$
B_{n}=\left(A_{n} \oplus S_{n}\right) \cup B_{n-1} \quad(n=1,2, \ldots, \mathrm{N})
$$

This method extends each target domain towards both ends, and thus connects the two adjacent crack fragments. The red circle mark on Figure 5(a) is one of the crack breaks after threshold segmentation, and Figure 5(d) is the crack fragment in the circle.

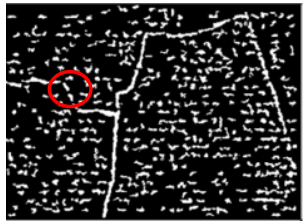

(a) Image after segmentation.

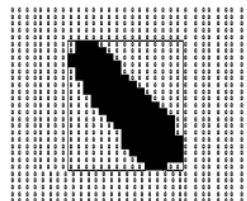

(d) Crack fragment in (a).

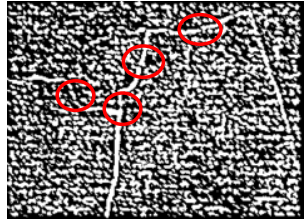

(b) Result of ordinary dilation

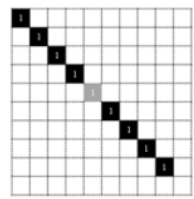

(e) Adaptive structure element.

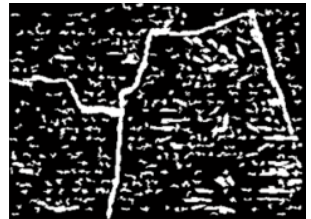

(c) Result of the proposed method.

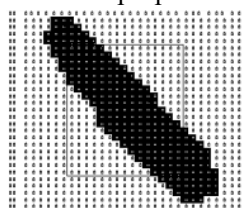

(f) Fragment after adaptive dilation.

Figure 5. Result of adaptive dilation based on crack direction:(a)-(c), (d)-(f).

Figure 5(e) shows the structural element that is adaptively constructed according to the angle of the fragment $\theta$. When Figure 5(d) is dilated with this structural element, the obtained image is dilated along the direction of $\theta$ and does not expand in other directions, as shown in Figure 5(f). Therefore, the dilated crack fragments can be connected to adjacent crack parts, making the crack more complete. Figure 5(b) is the result of common morphological dilation. It can be seen that the morphological operations are performed on each noise and crack fragment; however, there are still multiple crack breaks in the red circle mark. As shown in Figure 5(c), after the application of the method of adaptive dilation based on crack direction given in this paper, crack breaks are completely connected, and only the domains which conform to the crack characteristics have been dilated.

\subsection{Multiple filtering based on adaptive feature thresholds}

After dilation, the cracks are relatively complete, but it is necessary to further remove non-cracks by filtering. Filtering by using a feature threshold alone is not ideal. A multiple filtering method based on adaptive feature thresholds is proposed. According to the analysis of the results of the feature distributions in Section 2.1, four adaptive feature thresholds, namely $\mathrm{T}_{\mathrm{A}}, \mathrm{T}_{\mathrm{CV}}, \mathrm{T}_{\mathrm{COM}}$, and $\mathrm{T}_{\mathrm{L}}$, can be used for filtering, one after another. The process is as follows:

(1). Filtering based on the connected domain area. The image after adaptive dilation is denoted as $f_{0}(i, j)$, then the connected domain $C_{k}(x, y)$ can be extracted, the area of each domain is $A_{k}$, and the total number is $N_{A} . f_{0}(i, j)$ is filtered based on the area adaptive threshold $\mathrm{T}_{\mathrm{A}} . f_{1}(i, j)$ is obtained, as shown in Eq. (2).

$$
f_{1}(i, j)=\left\{\begin{array}{c}
0, f_{0}(i, j) \in C_{k}(x, y) \text { and } A_{k}>T_{A}, k=1,2 \ldots N_{A} \\
f_{0}(i, j), \text { otherwise }
\end{array}\right.
$$


(2). Filtering based on connected domain convexity. Extract all connected domains $D_{k}(x, y)$ from $f_{1}(i, j)$; the convexity of each domain is $C V_{k}$ and the total number is $N_{C V}$. The adaptive threshold $\mathrm{T}_{\mathrm{CV}}$ is used to filter $f_{1}(i, j) . f_{2}(i, j)$ is obtained, as in Eq. (3).

$$
f_{2}(i, j)=\left\{\begin{array}{c}
0, f_{1}(i, j) \in D_{k}(x, y) \text { and } C V_{k}>T_{C V}, k=1,2 \ldots N_{c v} \\
f_{1}(i, j), \text { otherwise }
\end{array}\right.
$$

(3). Filtering based on connected domain compactness. Extract connected domains $E_{k}(x, y)$ from $f_{2}(i, j)$; the compactness of each domain is $C O M_{k}$ and the total is $N_{\text {COM }}$. Use the adaptive threshold $\mathrm{T}_{\mathrm{COM}}$ to filter $f_{2}(i, j) . f_{3}(i, j)$ is obtained, as in Eq. (4).

$$
f_{3}(i, j)=\left\{\begin{aligned}
0, & f_{2}(i, j) \in E_{k}(x, y) \text { and } \operatorname{COM}_{k}>T_{\text {COM }}, k=1,2 \ldots N_{\text {COM }} \\
f_{2}(i, j), & \text { otherwise }
\end{aligned}\right.
$$

(4). Filtering based on connected domain max-distance. Extract connected domains $F_{k}(x, y)$ from $f_{3}(i, j)$; the maximum distance of each domain is $L_{k}$ and the total number is $N_{L}$. Use the adaptive threshold $\mathrm{T}_{\mathrm{L}}$ to filter $f_{3}(i, j) . f_{4}(i, j)$ is obtained, as in Eq. (5).

$$
f_{4}(i, j)=\left\{\begin{aligned}
0, & f_{3}(i, j) \in F_{k}(x, y) \text { and } L_{k}<T_{L}, k=1,2 \ldots N_{L} \\
f_{3}(i, j), & \text { otherwise }
\end{aligned}\right.
$$

The result of the four-stage adaptive morphological filtering is the final crack detection result, as shown in Figure 6. It can be seen that without modeling, the multiple filtering method in this paper can effectively filter out almost all noise and pseudo-cracks, and the cracks in the obtained image are complete and clear. Figure 7 shows the filtering result for an image with cracks without adaptive dilation based on the crack direction. It can be seen that the crack breaks are serious. Therefore, comparing Figure 6 and Figure 7, we can see that the adaptive dilation method proposed in this paper can effectively fill crack breaks and improve the crack integrity, and to a certain extent provide a guarantee for the accuracy of the multi-level filtering based on adaptive thresholds.

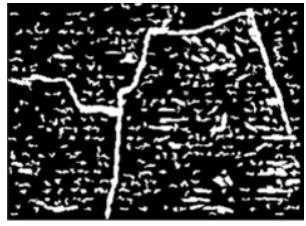

(a) After adaptive dilation.

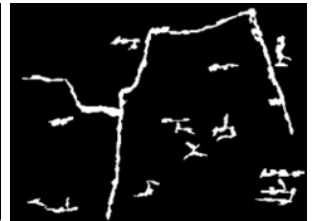

(d) Filtered by $\mathrm{T}_{\mathrm{A}}$.

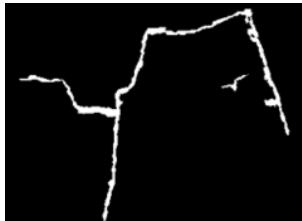

(d) Filtered by $\mathrm{T}_{\mathrm{CV}}$.

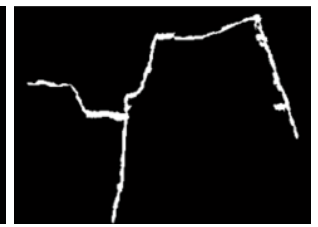

(d) Filtered by $\mathrm{T}_{\mathrm{COM}} \& \mathrm{~T}_{\mathrm{L}}$. Figure 6. Result of multiple adaptive feature threshold filtering:(a)-(d).

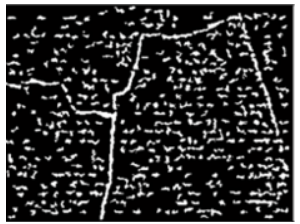

(a) Image without adaptive dilation.

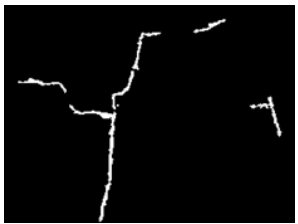

(b) Filtering result.

Figure 7. Result of multiple filtering based on adaptive feature thresholds without adaptive dilation:(a)-(b). 

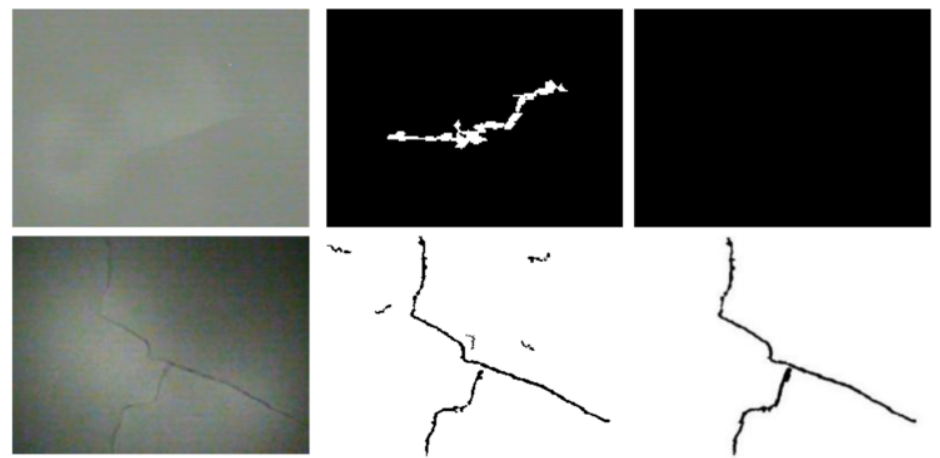

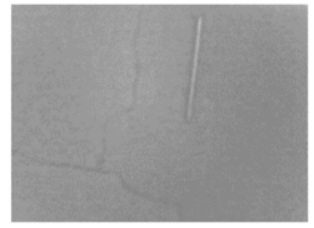

(a) Original infrared image.

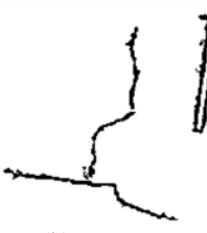

(b) SVDD method
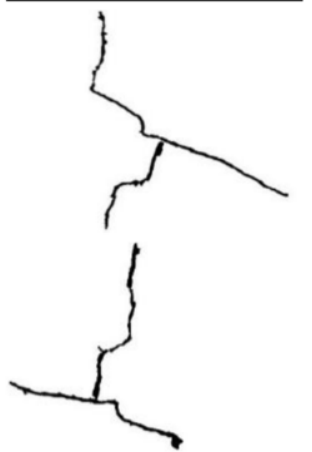

(c) The proposed method

Figure 8. Original infrared image and detection result.

The test results of this method and Support Vector Data Description (SVDD) [19] are compared in Figure 8. The crack image detected by SVDD usually has breaks, and there are still some noise and pseudo-cracks in the image, which need to be identified one by one to distinguish them from a real crack. Based on the proposed method, when there is no crack in the original image, the method can accurately detect no crack in the image. In the case of uneven illumination, the method can still accurately detect clear and complete crack images. A disturbing object on the concrete surface will also be filtered out, leaving only the crack image.

\section{Validity of the method}

Some quantitative indicators are used to evaluate the performance of the detection methods and the results of this paper [20]. For the crack image detection results, TP (true positives) is the number of crack images detected correctly, TN (true negatives) is the number of non-crack images detected correctly, FP (false positives) is the number of non-crack images detected as crack images, and FN (false negatives) is the number of crack images detected as non-crack images.

The test sample images were taken from laboratory concrete samples and bridge surfaces using the TC388 uncooled focal plane infrared sensor module with maximum supported resolution of $640 \times 480$ pixels, including 123 crack images and 43 non-crack images, with a ratio of $3: 1$. For the image processing and filtering method proposed in this paper, as shown in Figure 1, the results are shown in Table 1. For further comparative verification, we selected 43 infrared images with cracks and 43 images without cracks, and compared the results with those of the algorithm CrackIT [21-22] and SVDD [19] in recent years, as shown in Table 2.

Table 1. Detection performance

\begin{tabular}{cccccccc}
\hline TP & TN & FP & FN & Accuracy & Precision & Recall & FPR \\
\hline 121 & 40 & 3 & 2 & $96.99 \%$ & $97.58 \%$ & $98.37 \%$ & $6.98 \%$ \\
\hline
\end{tabular}


Table 2. Detection accuracy of the three algorithms

\begin{tabular}{ccc}
\hline Crack IT method & SVDD method & The proposed method \\
\hline $70.34 \%$ & $92.49 \%$ & $95.35 \%$ \\
\hline
\end{tabular}

Table 1 and Table 2 show that the proposed crack detection algorithm can not only filter out most of the interference and pseudo-cracks, but also has higher detection accuracy. In actual images, the detection accuracy of the CrackIT algorithm is not ideal due to uneven illumination, etc. In this paper, by using the difference of morphological characteristics between real cracks and noise and pseudo-cracks, the proposed adaptive morphological dilation and multiple filtering based on adaptive feature thresholds method can obtain and detect real cracks effectively, with a high detection accuracy of $95.35 \%$. This method has a fast processing speed and a detection time of $0.35 \mathrm{~s}$, which meets the requirements of real-time detection of concrete bridge cracks and is suitable for practical engineering applications.

\section{Conclusion}

This paper proposed a real-time crack detection method for bridge concrete based on infrared images. Generally, crack detection methods based on image processing often have the problem of crack breaks after threshold segmentation, resulting in low detection accuracy or incomplete cracks detected. To address this problem, an adaptive morphological dilation method based on crack direction is proposed in this paper, which can not only enhance the completeness of crack images, but also protect the crack edges to a certain extent. Most of the existing crack image detection methods attempt to achieve high detection accuracy by increasing the algorithm complexity, which is not suitable for application scenarios that require real-time detection. This paper proposed a detection method using multiple filtering based on adaptive feature thresholds, which can filter out almost all noise and pseudo-cracks, and finally detect complete real cracks without modeling. It is simple and reliable, with a detection accuracy that can reach $95.35 \%$. It has a fast processing speed and takes 0.35 seconds for detection, which meets the requirements of real-time processing and is suitable for practical engineering applications.

\section{References}

[1]. Zhang J, Wang FY, Wang K, et al. Data-Driven Intelligent Transportation Systems: A Survey. IEEE T. Intel. Transp. 2011, 12(4):1624-1639.

[2]. Miguel G, David B, Oscar M, Llorca DF, Sotelo MA. Adaptive road crack detection system by pavement classification. Sensor. Basel. 2011,11(10):9628-9657.

[3]. Feizi-Derakhshi, Mohammad-Reza, Azarafza. Computer modeling of crack propagation in concrete retaining walls: A case stud. Comput. Concrete. 2017,19(5):509-514.

[4]. Dai Y, Wang P, et al. Asphalt pavement pothole detection and segmentation based on wavelet energy field. Math. Prob. Eng. 2017(8), 1-13.

[5]. Zou Q, Cao Y, Li Q. CrackTree: automatic crack detection from pavement images. Pattern Recognition Letters, 2012, 33(3):227-238.

[6]. Su T, Yang M. Morphological segmentation based on edge detection-II for automatic concrete crack measurement. Computers \& Concrete. 2018,21(6):727-73.

[7]. Shi Y, Cui L, Qi Z, Fan M, Chen Z. Automatic road crack detection using random structured forests. IEEE Transactions on Intelligent Transportation Systems. 2016, 17(12): 3434-3445.

[8]. Zhao G, Wang T, Ye J. Anisotropic clustering on surfaces for crack extraction. Machine Vision \& Applications. 2015,26:675-688 
[9]. Jahanshahi MR, Masri SF, et al. An innovative methodology for detection and quantification of cracks through incorporation of depth perception. Machine Vision and Applications. 2013, 24(2):227-241.

[10]. Lee B Y, Kim Y, Yi S T, et al. Automated image processing technique for detecting and analyzing concrete surface cracks. Structure and Infrastructure Engineering. 2013, 9(6): 567-577.

[11]. Jang K, Kim N, An Y K. Deep learning-based autonomous concrete crack evaluation through hybrid image scanning. Structural Health Monitoring. 2019, 1475921718821719

[12]. Nguyen N T H, Le T H, Perry S, et al. Pavement Crack Detection using Convolutional Neural Network. ACM Association for Computing Machinery, Da Nang, Viet Nam, December.2018.

[13]. Ye H W, Duan X, Yang J C. Research on fatigue crack detection of steel bridge based on infrared thermal imaging. National Bridge Academic Conference. 2014,180-185.

[14]. Tashan J, Al-Mahaidi R. Detection of cracks in concrete strengthened with CFRP systems using infrared thermography. Composites Part B Engineering. 2014, 64:116-125.

[15]. Jang K, An Y K. Multiple crack evaluation on concrete using a line laser thermography scanning system. SMART STRUCTURES AND SYSTEMS. 2018, 22(2):201-207.

[16]. Xu Z, Zhao X, Song H, et al. Asphalt pavement crack recognition algorithm based on histogram estimation and shape analysis. CHINESE JOURNAL OF SCIENTIFIC. 2010(10):102-108.

[17]. $\mathrm{Xu} \mathrm{G}$, et al. A method for surface fracture extraction of structures based on the characteristics of multiple connected domains. Huazhong Univ.of Sci.\& Tech.(Natural Science Edition). 2019, 47(10):52-55+68.

[18]. Fujita Y, Hamamoto Y. A robust automatic crack detection method from noisy concrete surfaces. Machine Vision \& Applications. 2011, 22(2):245-254.

[19]. Lin W G, Sun Y C, Yang Q N, Lin Y R. Real-time comprehensive image processing system for detecting concrete bridges crack. Computers and Concrete. 2019,23 (6): 445-457.

[20]. Prasanna P, Dana K J, Gucunski N, et al. Automated crack detection on concrete bridges. IEEE T. Autom Sci. Eng. 2016, 13(2):591-599.

[21]. Oliveira H, Correia P L. Automatic road crack detection and characterization. IEEE T. Intell. Transp.2013,14(1):155-168.

[22]. Oliveira H, Correia P. CrackIT-an image processing toolbox for crack detection and characterization. IEEE International Conference on Image Processing, Paris, France, October. 2014. 